\title{
Treatment of Polymers by Plasma Immersion Ion Implantation for Space Applications
}

I. H. Tan', M. Ueda', R. S. Dallaqua', J. O. Rossi' , A. F. Beloto ${ }^{2}$ Instituto Nacional de Pesquisas Espaciais - ${ }^{1}$ Laboratório Associado de Plasma 2Laboratório Associado de Materiais e Sensores, São José dos Campos, SP, Brazil M. H. Tabacknics, Instituto de Física da USP, São Paulo, SP, Brazil

N. R. Demarquette

Escola Politécnica da USP - Depto. Eng. Metalúrgica e de Materiais, São Paulo, SP, Brazil Y. Inoue

Department of Materials Processing Engineering, Nagoya University, Chikusa, Nagoya, Japan

VIIth International Conference on Plasma-Based Ion Implantation (PBII) San Antonio, TX, USA 


\section{Outline}

- Motivation

- Objectives

- Direct Al implantation

- Recoil Al implantation

- Analysis

- Results

- Conclusions and Future Work 


\section{Motivation}

\section{Protection of Components for Spacecrafts Orbiting LEO}

Low Earth Orbit (LEO) environment (180-650 km ): rich in atomic oxygen, which degrades polymeric materials (such as Kapton, Mylar or Teflon) used in satellites.

May erode certain polymers by over $2 \mu \mathrm{m}$ in 90 days

Oxygen resistant polymers could improve the lifetime of satellites and space stations and could find many applications in space, including huge fold-up antennas, inflatable mirrors \& lenses, solar sails...

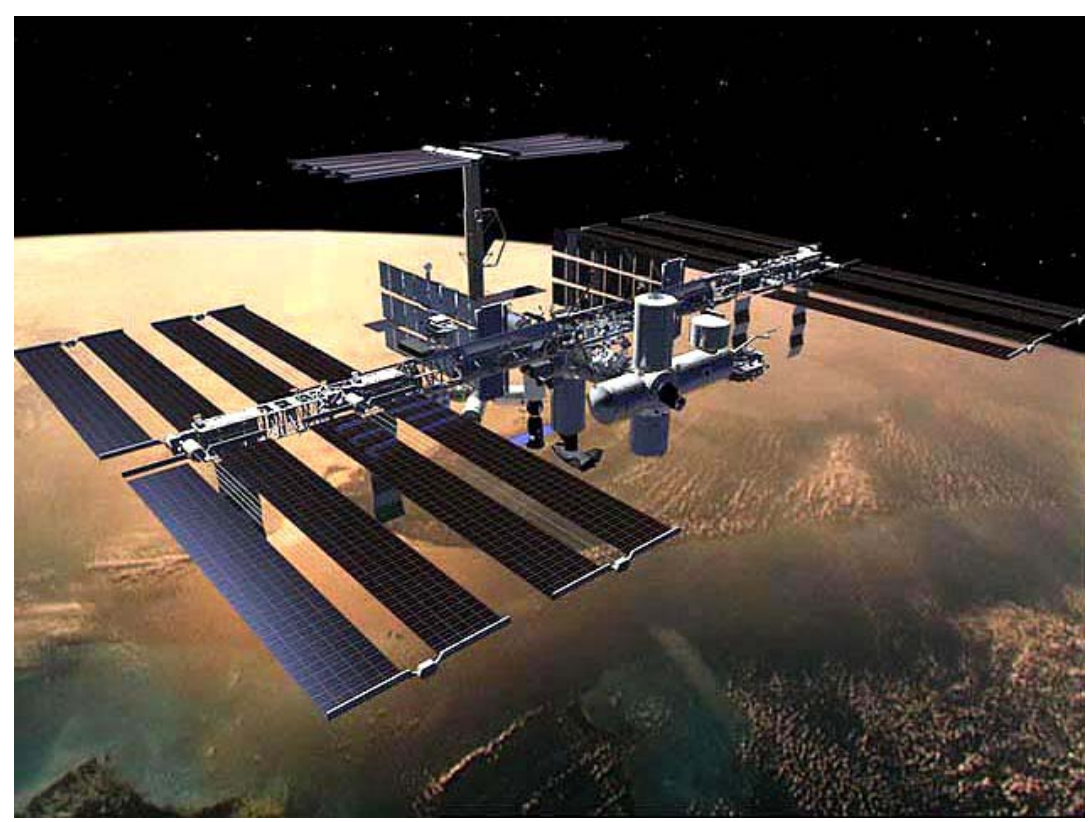

International Space Station orbiting LEO region (450 km) 


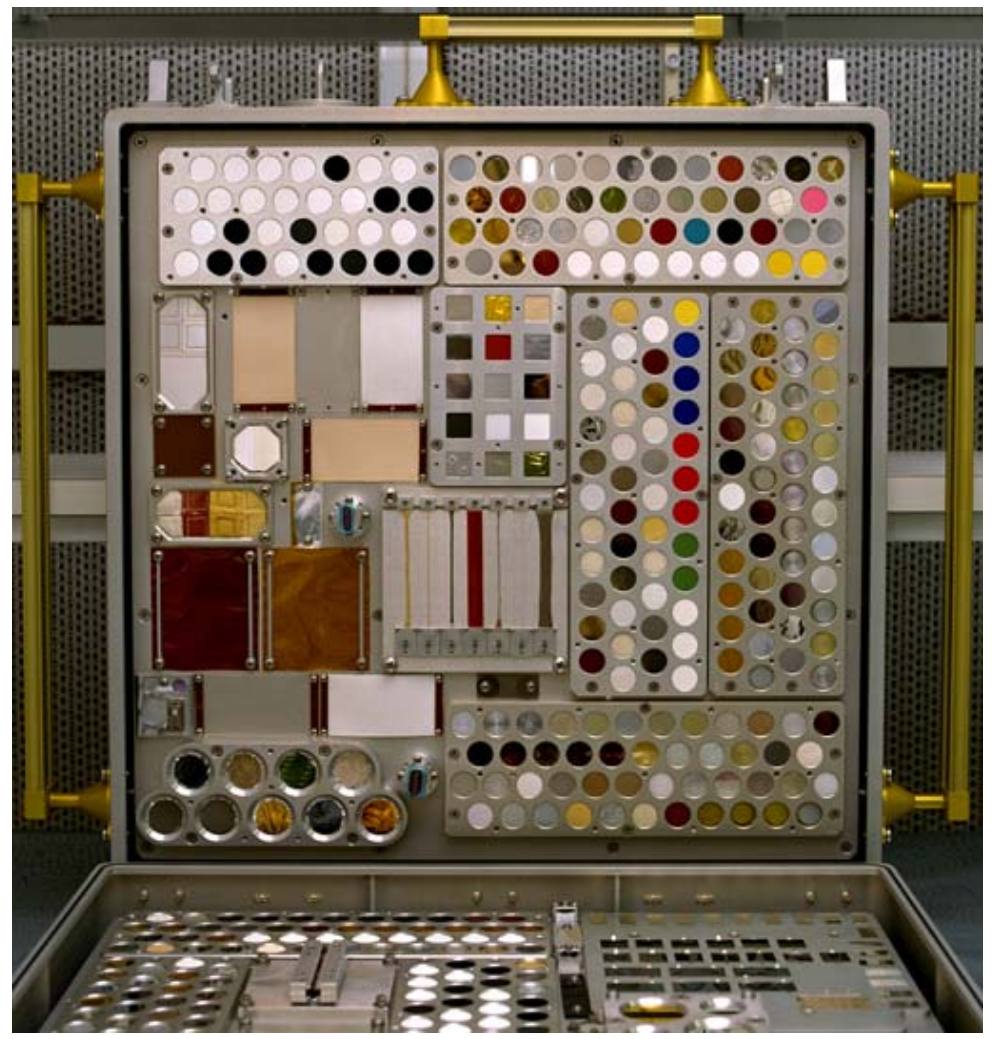

Materials International Space Station

Experiment - MISSE project

Trays of materials samples will expose 750 materials to LEO environment, for 18 months.

Oxidation protection: Thin layers of several metal oxides such as $\mathrm{Al}_{2} \mathrm{O}_{3}, \mathrm{MgO}$, or $\mathrm{SiO}_{2}$ are being studied as protective coatings for polymers in LEO

Thermal transients $\left(-100^{\circ} \mathrm{C}\right.$ to $\left.+100^{\circ} \mathrm{C}\right)$ : Superior adhesion of the thin film is required $\rightarrow$ Metal Plasma Immersion Ion Implantation and Deposition 
PIII in polymers: charging of the dielectric is proportional to plasma density Typically for $\sim 20 \mu \mathrm{m}$ thick polymers:

$$
\begin{array}{ll}
\mathrm{n} \sim 10^{17} \mathrm{~m}^{-3} \rightarrow \Delta \mathrm{V} \sim 7 \mathrm{kV} & \text { in } 2 \mu \mathrm{s} \\
\mathrm{n} \sim 10^{15} \mathrm{~m}^{-3} \rightarrow \Delta \mathrm{V} \sim 700 \mathrm{~V} & \text { in } 60 \mu \mathrm{s}
\end{array}
$$

In metal plasmas generated by vacuum arcs:

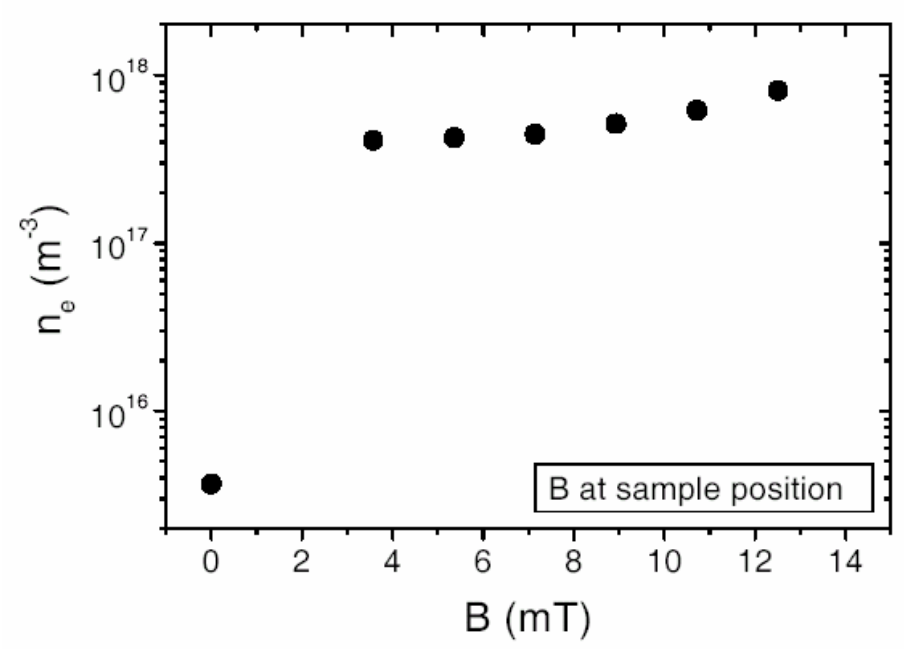

Magnetic field increases plasma density by two orders of magnitude 


\section{Objectives}

Aluminum implantation in Kapton ${ }^{\circledR}$ by three different methods

- Direct implantation in a magnetized Al plasma

- Direct implantation in an unmagnetized Al plasma

- Al deposition + implantation in nitrogen plasma (recoil implantation)

Resistance tests for space environment

- Oxygen degradation (oxygen plasmas)

- Thermal cycling

- Adhesion test 


\section{Direct Aluminum implantation}

Vacuum Arc Plasmas: HV trigger: arc initiation

Plasma drift velocity $\sim 10^{4} \mathrm{~m} / \mathrm{s}$

$$
\begin{aligned}
& \mathrm{I}_{\mathrm{arc}} \sim(100-1 \mathrm{k}) \mathrm{A} / 50 \mathrm{~V} \\
& \mathrm{I}_{\text {ion }} \sim \text { up to }(5-10) \% \mathrm{I}_{\mathrm{arc}}
\end{aligned}
$$

Macroparticle Filtering systems:

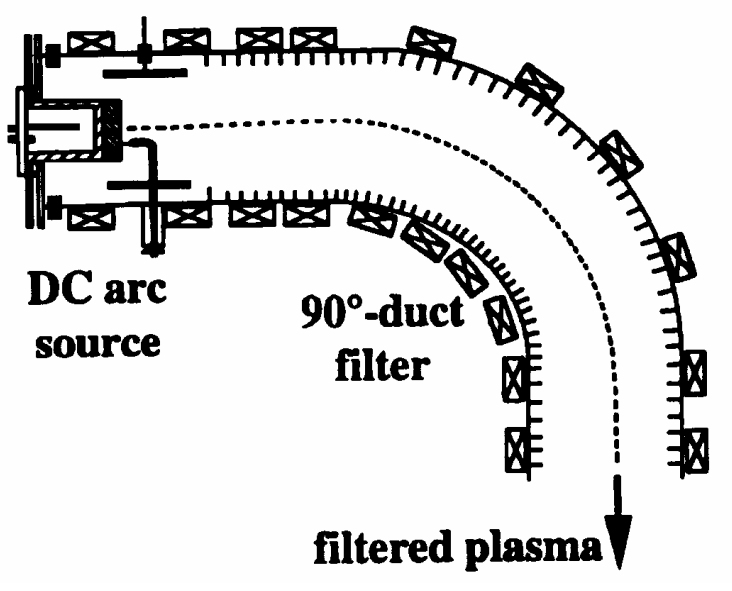

Curved magnetic filter

good filtering

not so good plasma transport

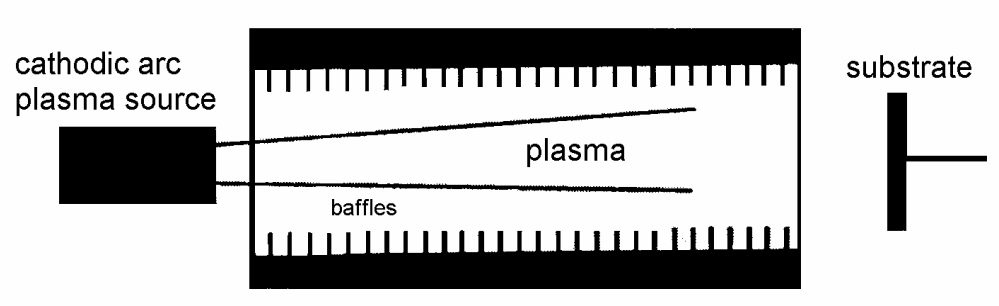

Straight magnetic filter

not so good filtering good plasma transport

Macroparticles avoided and deposition minimized by orienting samples parallel to plasma stream 


\section{Experimental Set-up}

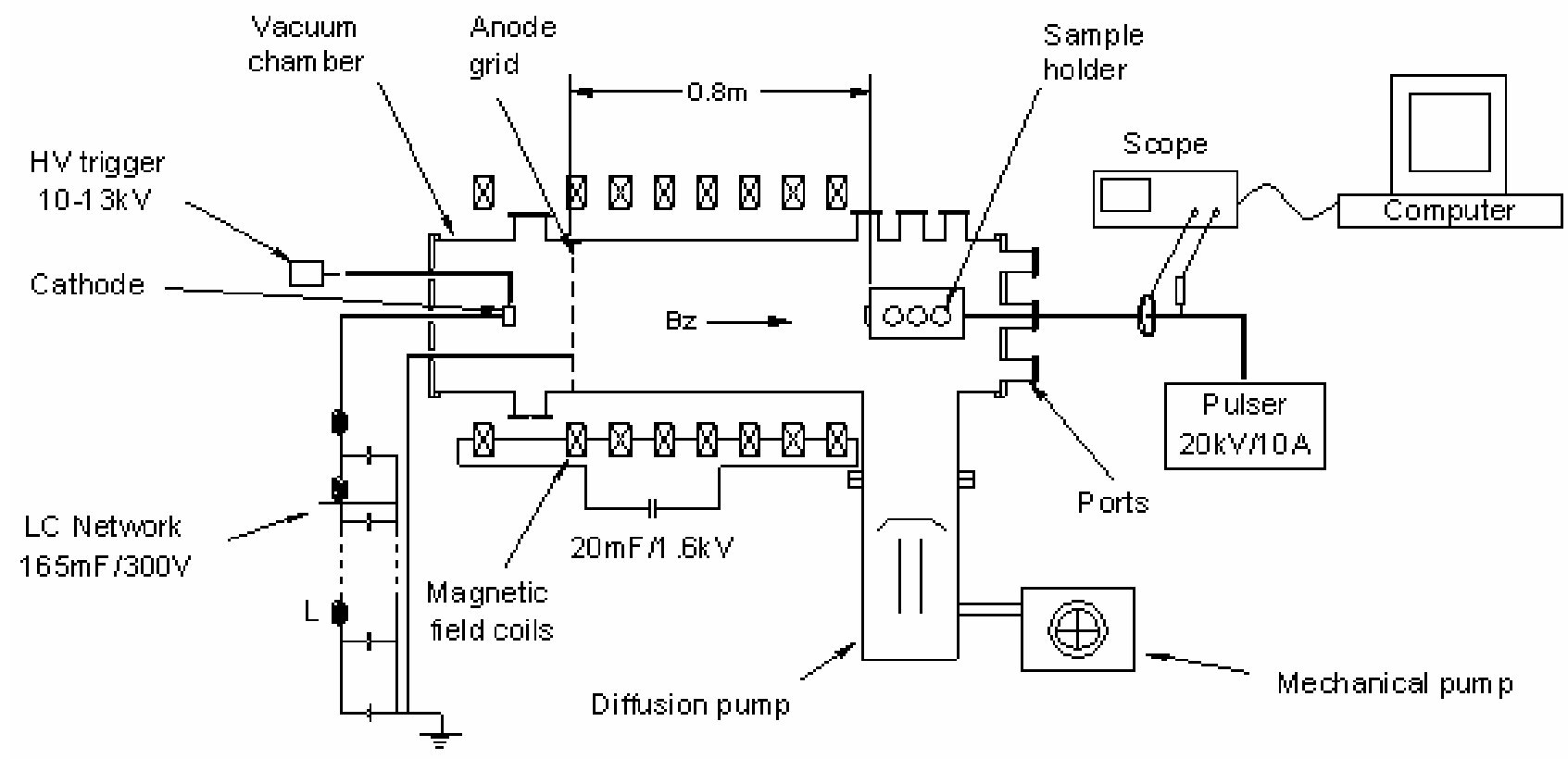

Vacuum arc:

Vacuum chamber

Sample holder:
Al cathode Tungsten grid anode HV trigger: $10-13 \mathrm{kV}$ $\phi=0.22 \mathrm{~m}, \mathrm{~L}=1.05 \mathrm{~m}$

Base pressure $\sim 1 \times 10^{-4} \mathrm{~Pa}$ B field: 150G-7kG $85 \mathrm{~cm}$ from cathode 
- Implantation Conditions
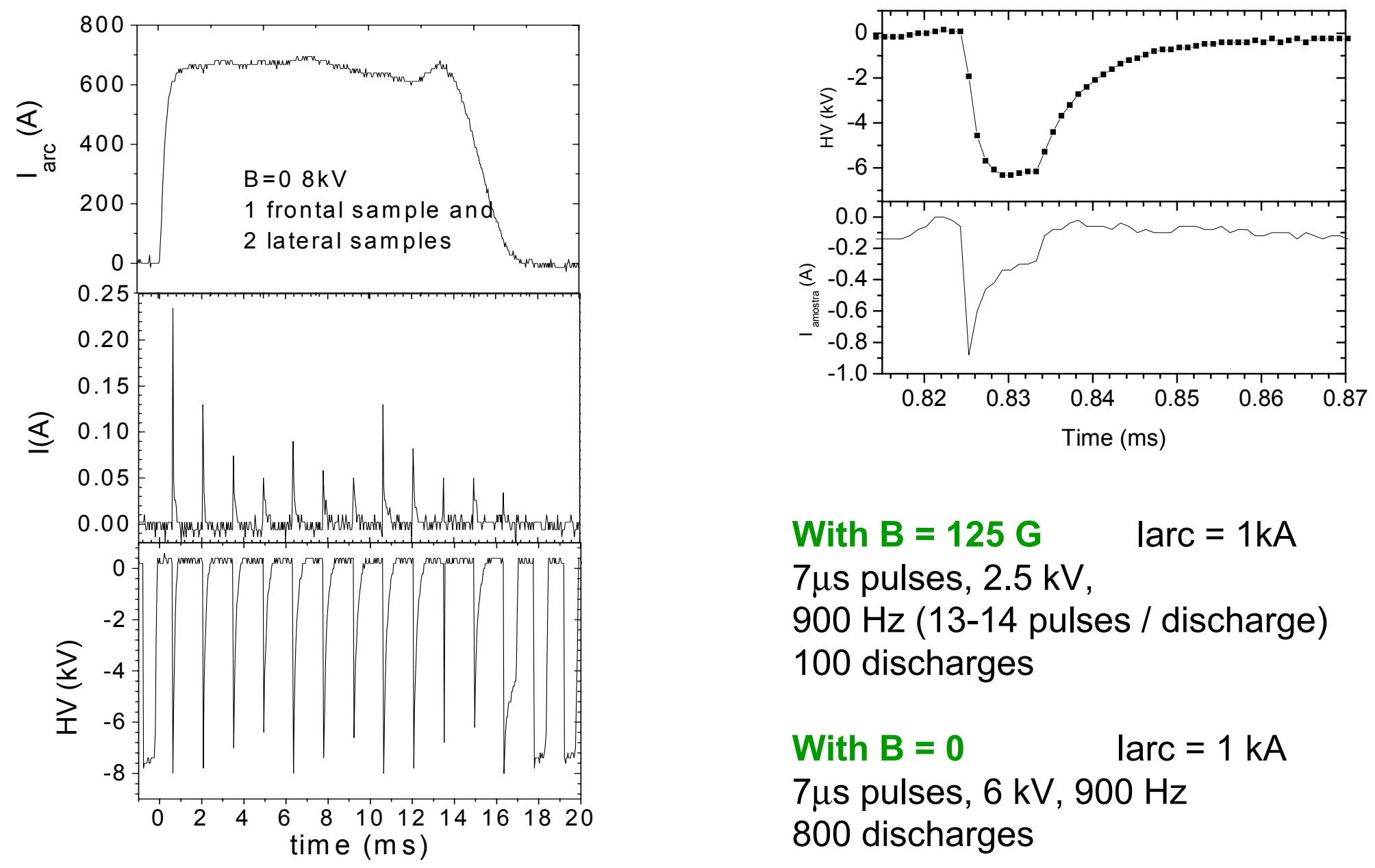

With $B=125 \mathrm{G} \quad \operatorname{larc}=1 \mathrm{kA}$

$7 \mu \mathrm{s}$ pulses, $2.5 \mathrm{kV}$, $900 \mathrm{~Hz}$ (13-14 pulses / discharge) 100 discharges

With $\mathrm{B}=0 \quad \operatorname{larc}=1 \mathrm{kA}$

$7 \mu$ s pulses, $6 \mathrm{kV}, 900 \mathrm{~Hz}$ 800 discharges 


\section{Recoil Aluminum implantation}

$200 \AA$ aluminum film deposited by electron beam on a Kapton sample $(8.5 \mathrm{~cm} \times 12 \mathrm{~cm})$ wound around sample holder. HV in contact with deposited film.

Implantation in Nitrogen plasmas: $\mathrm{n} \sim 10^{10} \mathrm{~cm}^{-3}, \mathrm{~T}_{\mathrm{e}}<10 \mathrm{eV}$ HV pulses: $5 \mu \mathrm{s}, 100 \mathrm{~Hz}, 5 \mathrm{kV}$ treatment time of 30 minutes

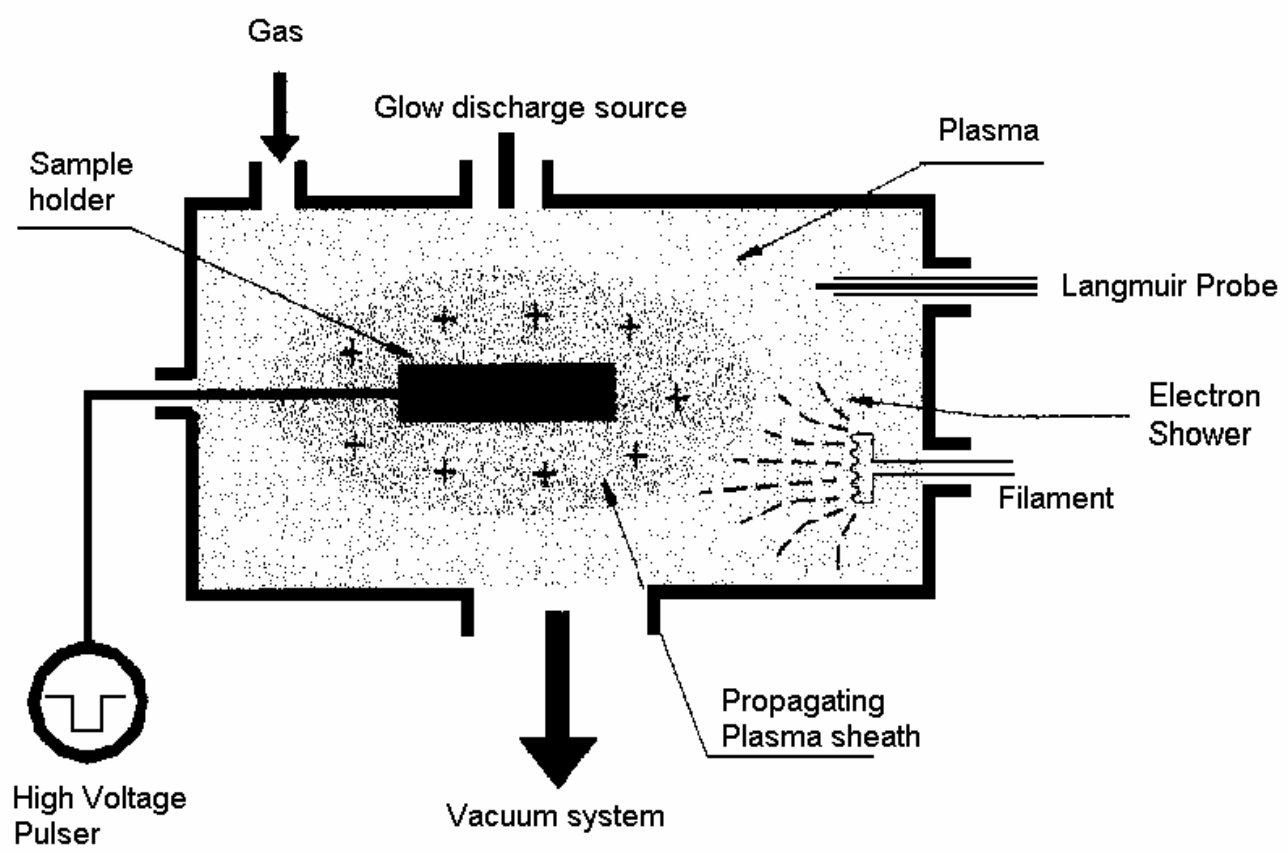




\section{Analysis}

\section{Elemental composition and morphology}

- $\mathrm{RBS}$

- XPS

- SEM , EDS

\section{Oxygen degradation}

- Oxygen plasma: $40 \mathrm{kHz}$ parallel plate capacitive reactor 200 mTorr, $200 \mathrm{~W} \rightarrow \mathrm{n} \sim 10^{10} \mathrm{~cm}^{-3}, \mathrm{~T}_{\mathrm{e}} \sim(1-2) \mathrm{eV}$

one hour exposure

\section{Thermal cycling}

- 1 minute liquid nitrogen immersion $\left(-196{ }^{\circ} \mathrm{C}\right)$

- 1 minute pre-heated oven $\left(100^{\circ} \mathrm{C}\right)$

- 15 cycles

\section{Adhesion Test}

- applying and removing a pressure sensitive tape + SEM 


\section{Results}

\section{Oxygen Degradation}

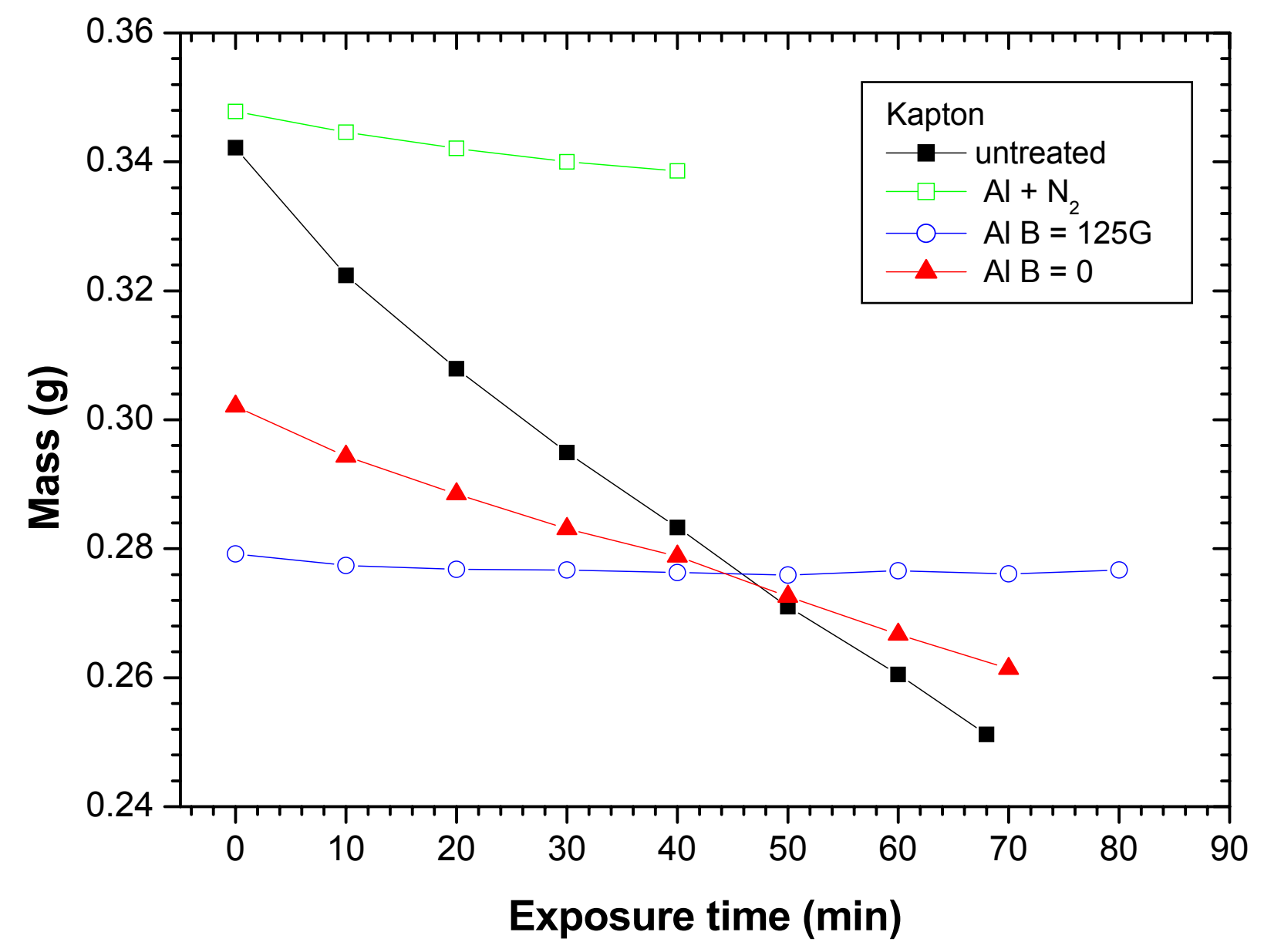


Kapton sample implanted with $\mathrm{Al}$ in a magnetized plasma

- $\quad$ RBS $\rightarrow$ retained doses of $10^{16}$ atoms $/ \mathrm{cm}^{2}$, but mostly at the surface

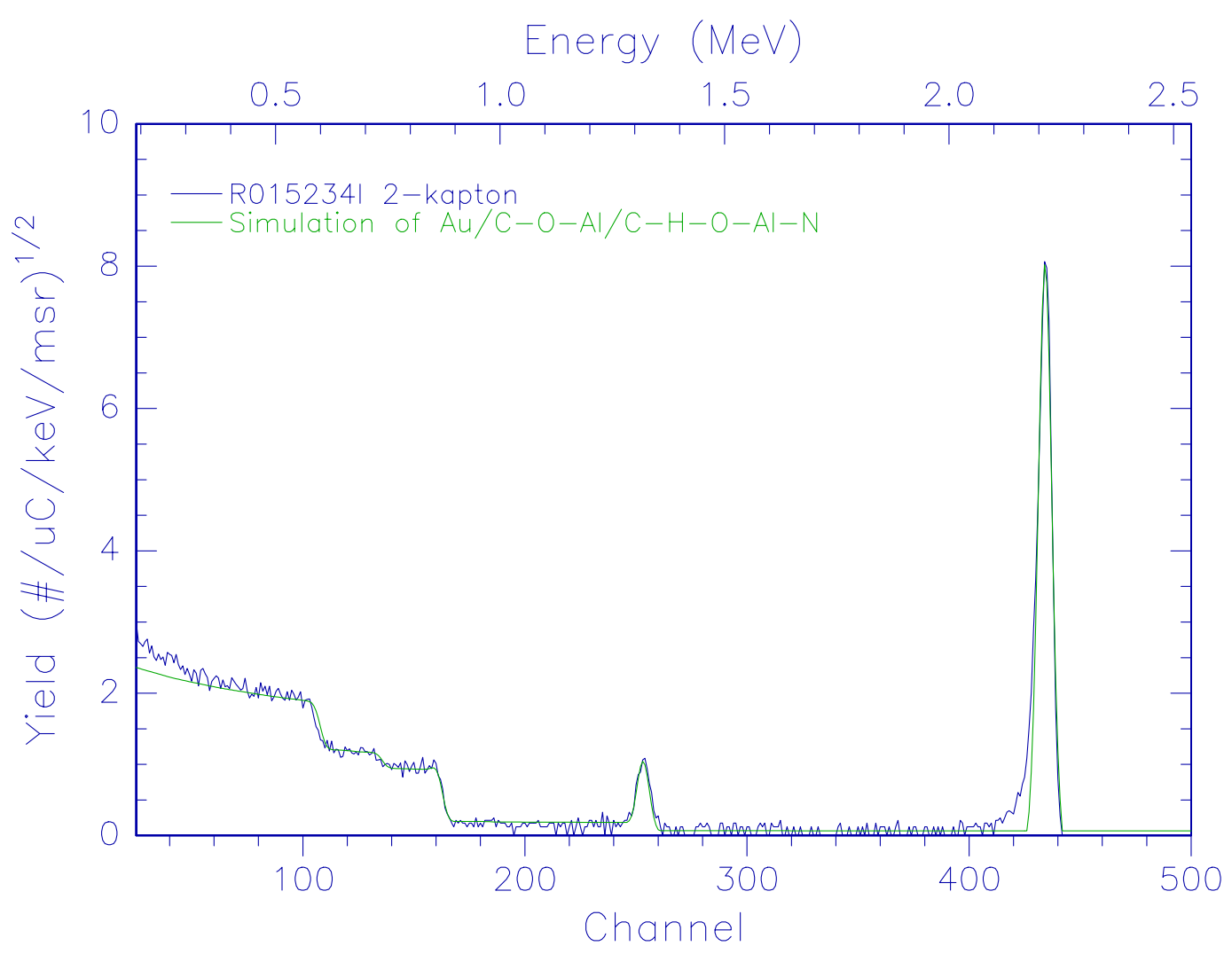


- $\mathbf{X P S} \rightarrow$ formation of an ion mixing layer

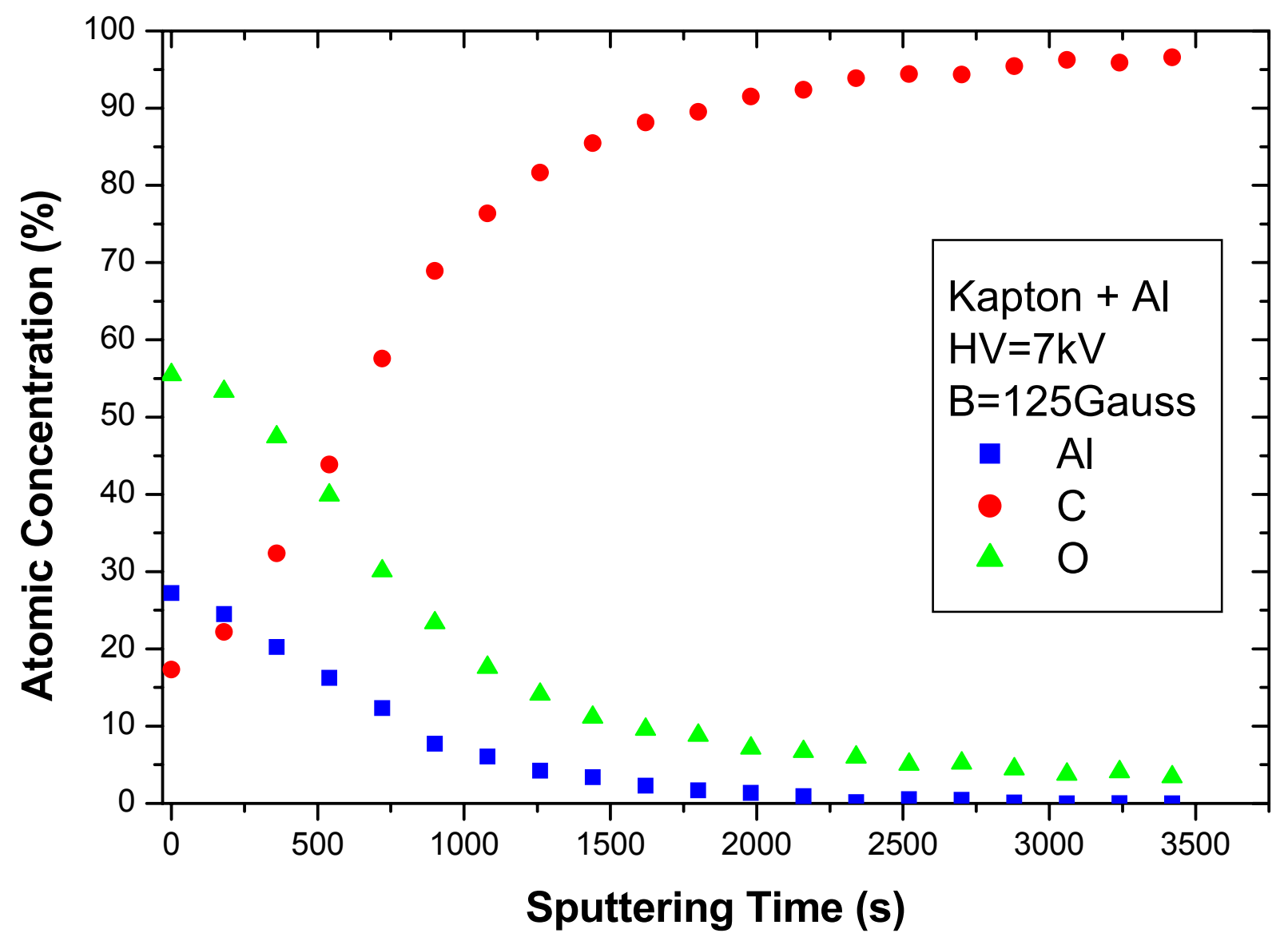


Kapton sample implanted with $\mathrm{Al}$ in a magnetized plasma

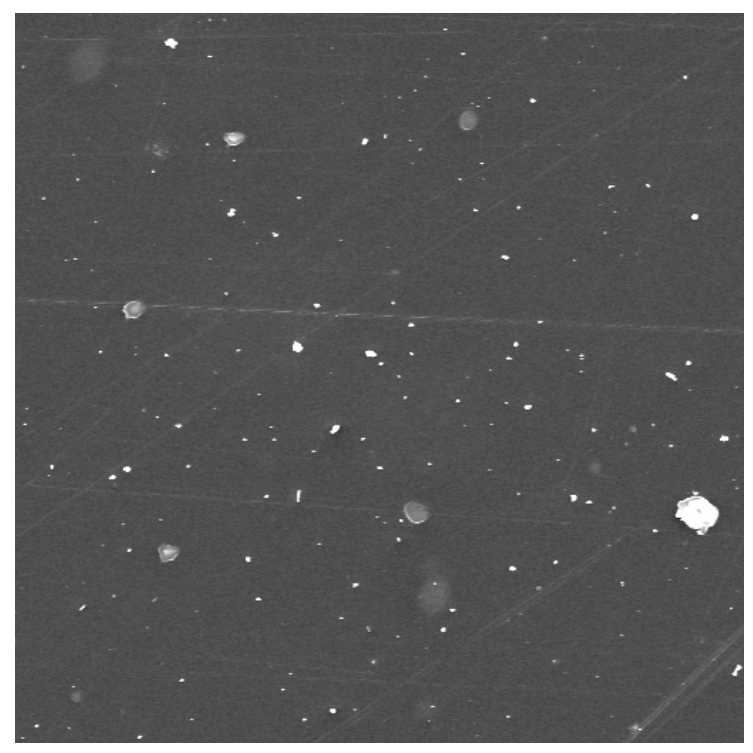

Without treatment after oxygen exposure $\rightarrow$

Without treatment

Treated sample after oxygen exposure + thermal cycling $\rightarrow$ + adhesion tests
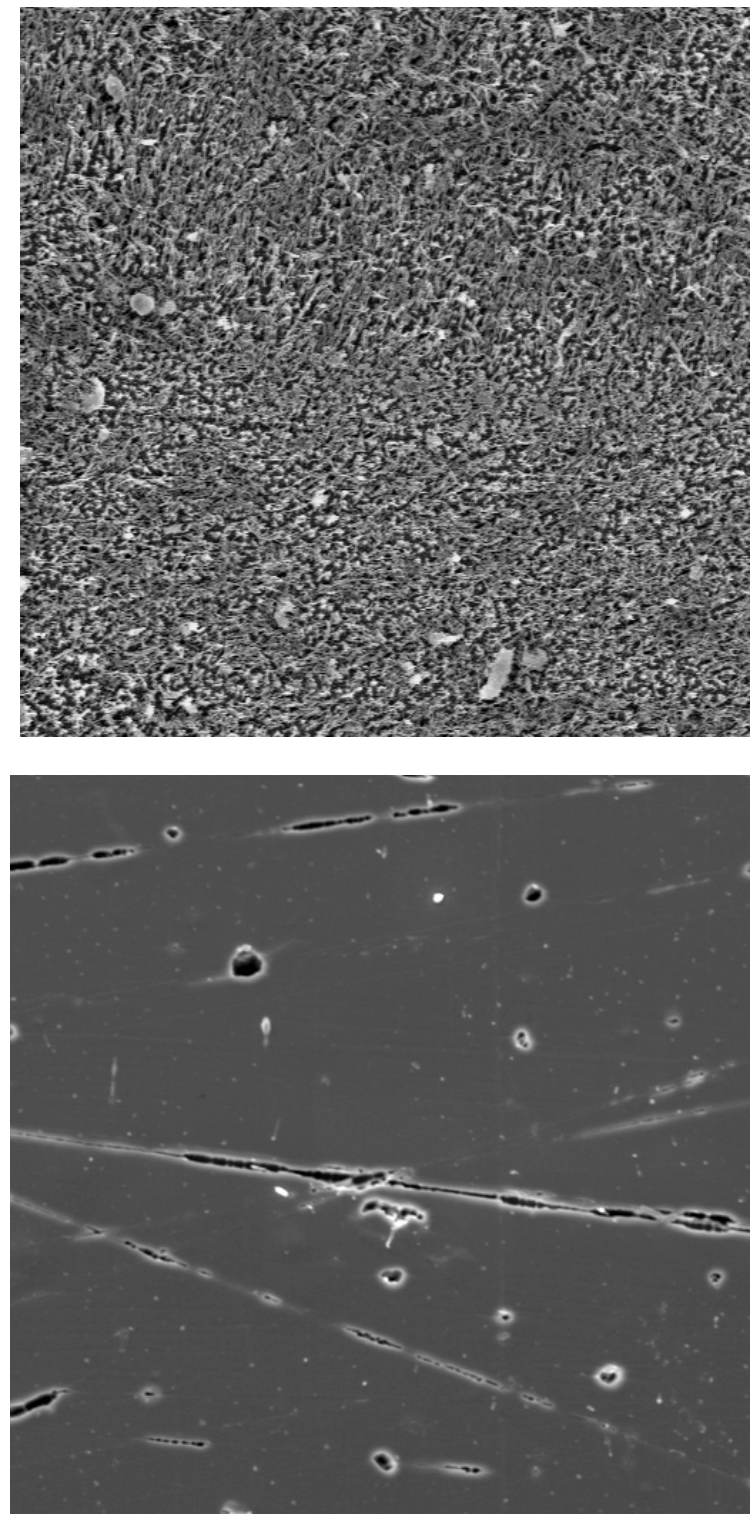
Kapton sample implanted with $\mathrm{Al}$ in an unmagnetized plasma

- Not uniform : good parts behave like magnetized case after exposure to O plasmas bad parts behave like untreated case after exposure to $O$ plasmas

- Possible causes: Insufficient dose misalignment with plasma stream

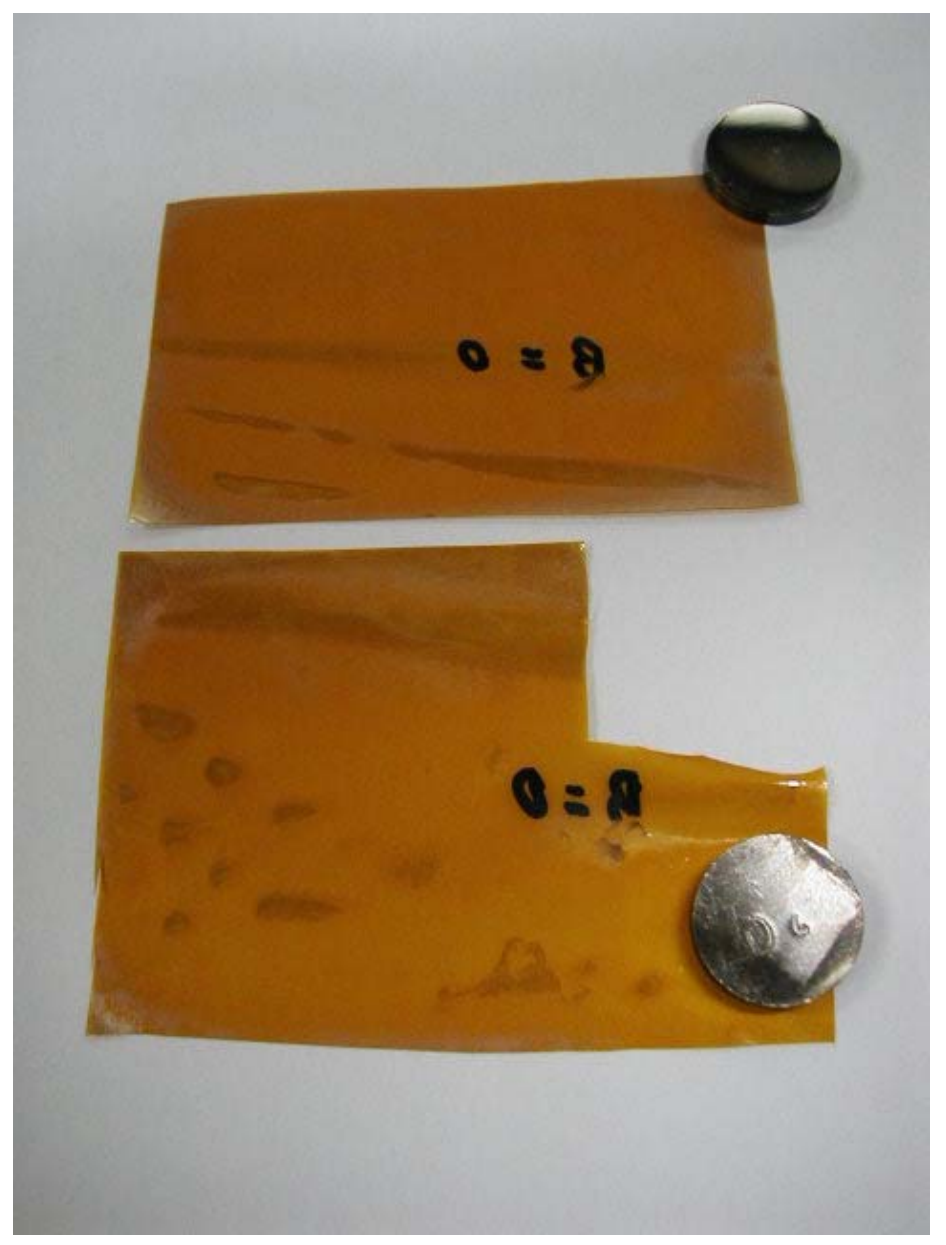


- $\quad$ RBS - "good" parts - about $100 \AA$ Al deposition mostly at surface

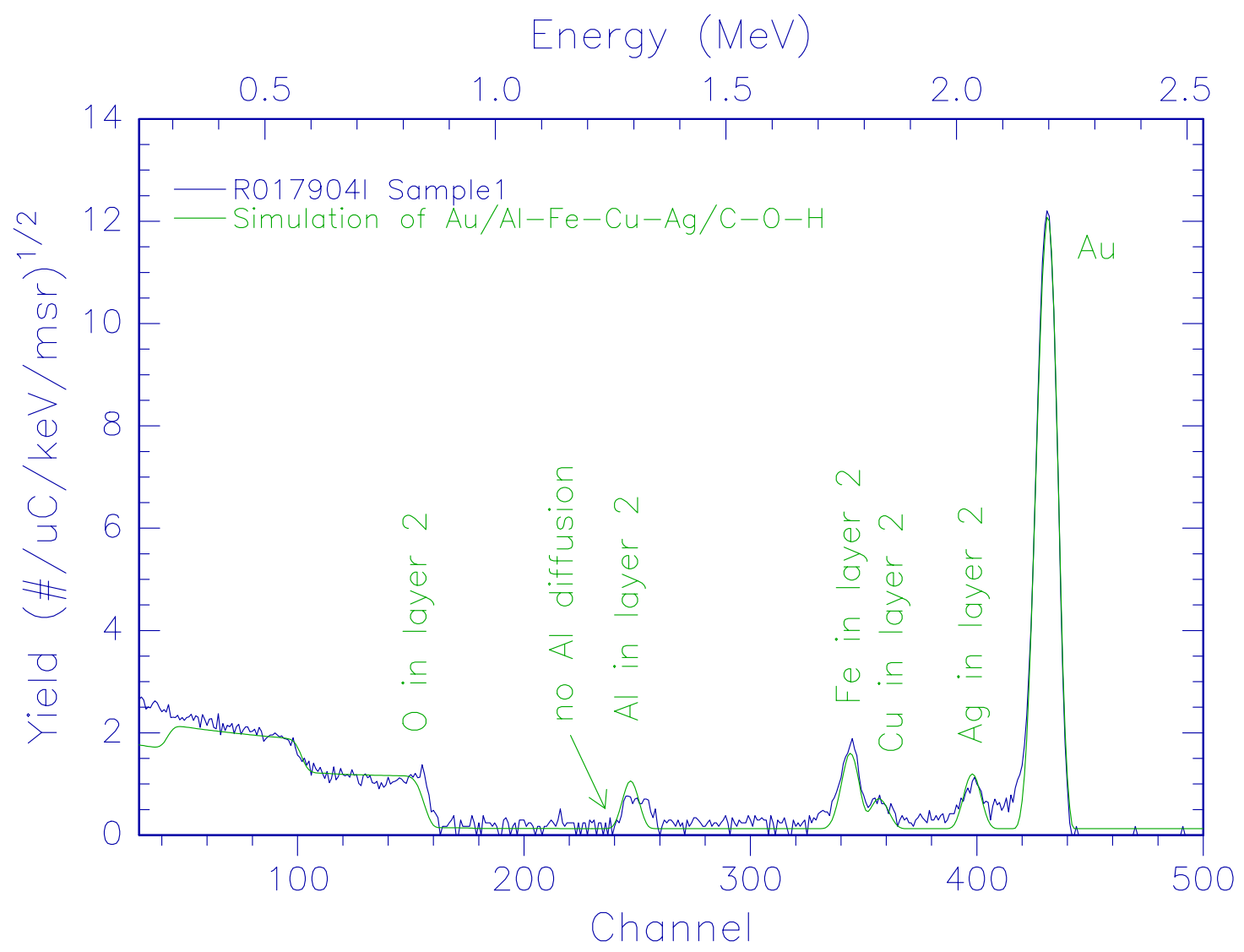

- Adhesion tests after thermal cycling showed no delamination 
- Kapton sample with Al deposition $(200 \AA)+$ nitrogen plasma implantation

- Formation of cracked film $\rightarrow$ probably stressed aluminum nitride

- Loss of transparency
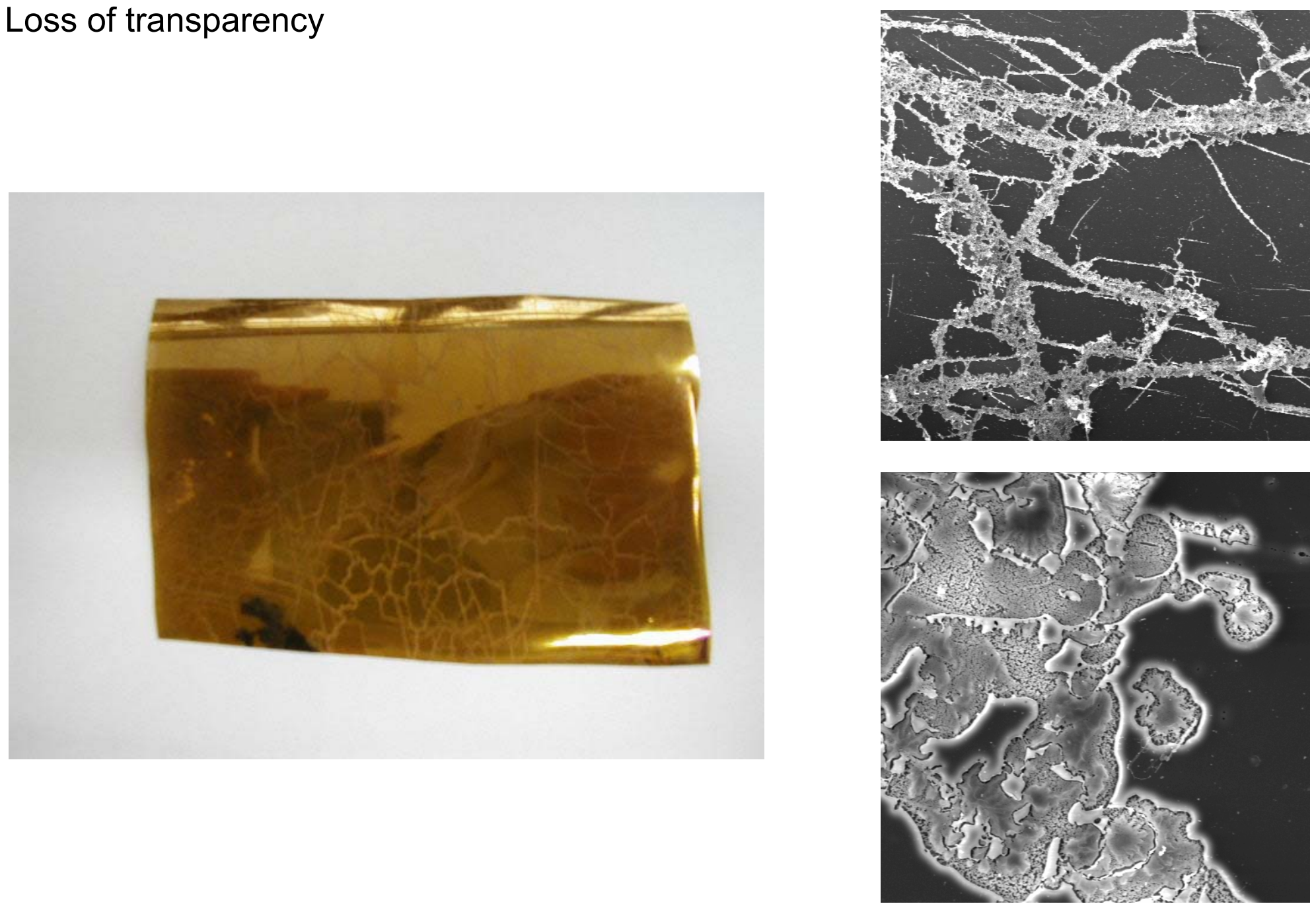
- $\quad$ RBS - Al deposited mostly at surface

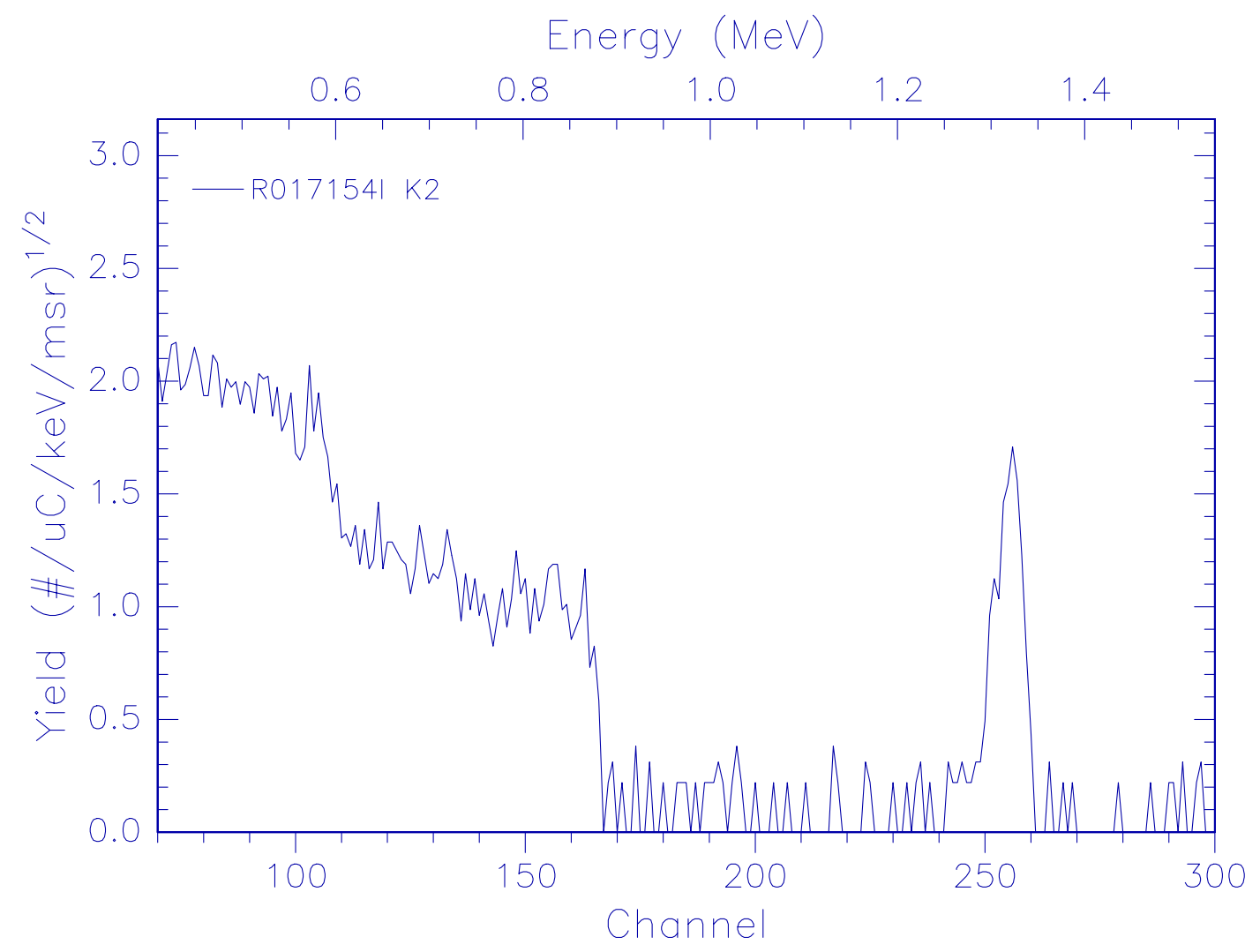

- Adhesion tests after thermal cycling showed no delamination 


\section{Conclusions}

- Kapton samples implanted with Al in a magnetized vacuum arc discharge resulted in excellent protection of the polymer against oxygen degradation.

- Retained doses of $10^{16}$ atoms $/ \mathrm{cm}^{2}$ were obtained, and although most of the atoms are concentrated on the surface, an intermediate ion mixing layer was formed.

- Adhesion test after thermal cycling shows good adhesion to the substrate.

- Implantation with Al in non-magnetized plasmas needs much longer treatment times, incompatible with present machine configuration.

- Al deposition by e-beam, followed by recoil implantation in a nitrogen plasma resulted in a cracked film, probably due to the formation of a stressed aluminum nitride film. Recoil implantation in an argon plasma is underway. 


\section{Acknowledgements:}

This work was supported by FAPESP (State of S.Paulo Research Funding Agency) 